\title{
Validity of Trunk Acceleration Measurement With a Chest-worn Monitor for Assessment of Exercise Intensity
}

Masahiko Mukaino ( $\nabla$ mmukaino@fujita-hu.ac.jp )

Fujita Health University

\section{Takayuki Ogasawara}

NTT Corporation

\section{Hirotaka Matsuura}

Fujita Health University

\section{Yasushi Aoshima}

Fujita Health University Hospital

\section{Takuya Suzuki}

Fujita Health University Hospital

\section{Masumi Yamaguchi}

NTT Corporation

Hiroshi Nakashima

NTT Corporation

\section{Eiichi Saitoh}

Fujita Health University

\section{Shingo Tsukada}

NTT Corporation

\section{Yohei Otaka}

Fujita Health University

\section{Research Article}

Keywords: Smart clothing system, Acceleration, Physical activity

Posted Date: December 10th, 2021

DOI: https://doi.org/10.21203/rs.3.rs-1148576/v1

License: (c) (1) This work is licensed under a Creative Commons Attribution 4.0 International License.

Read Full License 


\section{Abstract}

Background: Recent advancements in wearable technology has enabled easy measurement of daily activities, which can be applied in rehabilitation practice for the purposes such as maintaining and increasing the activity levels of the patients. A smart clothing system is one of the newly developed wearable systems that enables the measurement of physical activity such as heart rate and/or acceleration. In this study, we aimed to examine the validity of trunk acceleration measurement using a smart clothing system ('hitoe'system) in assessing the physical activity, which was measured using the expiratory gas analysis.

Methods: Twelve healthy individuals participated in the study. The trunk acceleration was simultaneously measured using a triaxial accelerometer embedded in a smart clothing activity monitoring system ('hitoe' system), and the percent $\mathrm{VO}_{2}$ reserve $\left(\% \mathrm{VO}_{2} \mathrm{R}\right)$ was determined by performing expiratory gas analysis during treadmill testing. Three parameters, that is, moving average (MA), moving standard deviation (MSD), and moving root mean square (RMS), were calculated using the norm of the trunk acceleration. The relationships between these accelerometer-based parameters and $\% \mathrm{VO}_{2} \mathrm{R}$ from expiratory gas analysis for each individual were examined.

Results: The values of MA, MSD, RMS, and $\% \mathrm{VO}_{2} \mathrm{R}$ were significantly different between levels $1,2,3$, and 4 in the Bruce protocol $(P<0.01)$. The average coefficients of determination for individual regression for $\% \mathrm{VO}_{2} \mathrm{R}$ vs. $\mathrm{MA}, \% \mathrm{VO}_{2} \mathrm{R}$ vs. $\mathrm{MSD}$, and $\% \mathrm{VO}_{2} \mathrm{R}$ vs. RMS were $0.89 \pm 0.05,0.96 \pm 0.03$ and $0.91 \pm 0.05$, respectively. The parameters based on the trunk acceleration measurements were significantly correlated with $\% \mathrm{VO}_{2} \mathrm{R}$ and activity levels. Among the parameters examined, MSD showed the best correlation with $\% \mathrm{VO}_{2} \mathrm{R}$, indicating high validity of the parameter for assessing physical activity.

Conclusions: The present results support the validity of the MSD calculated from the trunk acceleration measured with a smart clothing system in assessing the exercise intensity.

Trial registration: UMIN000034967

Registered 21 November 2018 (retrospectively registered).

\section{Background}

Recently, there have been several reports on activity monitoring using wearable devices, along with the dynamic development in measurement technology. Accelerometry is one of the main measurement modalities used for monitoring physical activity. A number of studies have reported its usefulness in monitoring the movements of individuals using devices, such as pedometers, which are worn on the waist and used for measuring step counts [1, 2] and wrist-worn type accelerometers [3, 4], which are increasingly becoming common these days. 
Given the increasing need for rehabilitation clinical practices to quantify daily activities of patients for the purpose of maintaining and increasing their activity levels, the use of an accelerometer would be beneficial in the assessment of the activity quantification of patients in rehabilitation. However, there may still be some difficulties in applying these devices to patients with motor impairments such as post-stroke paresis, which is frequently observed in the rehabilitation practice. For example, patients with paresis or lower limb injuries frequently use handrails or canes, which may interfere with accurate measurements using a wrist-worn accelerometer [5]. Measuring steps with an accelerometer can be inaccurate for patients with neurological disorders [6] due to reasons such as irregular step patterns and low gait speeds.

Therefore, there is a need for an alternative methodology optimized for activity quantification in patients with motor impairment.

Trunk acceleration measurement may be an option. The measurement of trunk movement should be less influenced by the upper and lower limb motions of patients with motor impairment and thus can be effective in quantifying the physical activity of the patients. The measurement of trunk acceleration has been used in activity monitoring in various manners. For example, there are several studies that evaluated gait parameters such as steps and asymmetry with trunk acceleration [7-9]. The chest-mounted accelerometer is also used for activity recognition [10,11] and fall detection [12]. However, the usability of trunk acceleration with a chest-worn monitor for the quantification of activity is not well investigated.

In addition, there may be a need for new index for the evaluation of the physical activity using accelerometer; to date, the use of indices such as step counts and device-specific activity counts, which are count of movement above a certain pre-set threshold has been common. However, the intensity of the activity should also be taken into consideration while assessing patients with motor impairment because the slower and smaller movement in daily activity of patients with motor impairment may not be sufficiently detected by the thresholds, which were set based on the activity of healthy subjects. Although the intensity of physical activity is usually defined by the oxygen consumption or heart rate, which reflects the oxygen supply for the activity, objective physical indices based on acceleration measurements that reflect the intensity should also be useful in assessing the character of the activity of patients with motor impairments.

Therefore, in this study, we investigated the validity of several indices based on trunk acceleration measurements for the assessment of the intensity of physical activity, in comparison with the exercise intensity determined via expiratory gas analysis. A smart clothing system, which is one of the emerging wearable monitoring systems, was used for measurement.

\section{Materials And Methods \\ 2.1 Participants}


Fourteen healthy adults ( 10 males; mean age of $29 \pm 5$ years) with no medical history of neurological, musculoskeletal, cardiovascular, or respiratory diseases participated in this study. Individuals who received medication that could potentially affect performance were excluded.

This study complied with the principles of the Declaration of Helsinki and was approved by the Medical Ethics Committee of Fujita Health University. All the participants provided written informed consent prior to participation.

\subsection{Procedures}

Each participant underwent treadmill testing following the Bruce protocol [13]. Respiratory gas analysis during exercise testing was performed using a mobile aerosol monitor (AE-100i, Medical Science, Tokyo, Japan). Participants were asked to avoid any high-intensity exercise and alcohol or caffeine consumption $24 \mathrm{~h}$ prior to the assessment. Before these tests, the resting $\mathrm{VO}_{2}$ while sitting was measured. The monitor was calibrated before and after each testing session using verified calibration gases. According to the Bruce protocol, participants started exercising at level 1 with a treadmill speed of $2.7 \mathrm{~km} / \mathrm{h}$ and an incline of $10 \%$ gradient for $3 \mathrm{~min}$. The speed and inclination were subsequently increased at 3 -min periods in the following manner: level $2,12 \%$ incline at $4.0 \mathrm{~km} / \mathrm{h}$; level 3,14\% incline at $5.5 \mathrm{~km} / \mathrm{h}$; level $4,16 \%$ incline at $6.8 \mathrm{~km} / \mathrm{h}$; level $5,18 \%$ incline at $8.1 \mathrm{~km} / \mathrm{h}$; level $6,20 \%$ incline at $8.9 \mathrm{~km} / \mathrm{h}$; and level 7, 22\% incline at 9.7 $\mathrm{km} / \mathrm{h}$. We considered $\mathrm{VO}_{2}$ to have reached the maximum value if the participants satisfied at least three of the following four criteria: (1) maximum voluntary exhaustion, as measured by the Borg CR-10 scale; (2) presence of a heart rate plateau ( $\Delta \mathrm{HR}$ between two consecutive work rates $\leq 4$ beats $\left.\cdot \mathrm{min}^{-1}\right)$; (3) presence of a $\mathrm{VO}_{2}$ plateau $\left(\Delta \mathrm{VO}_{2}\right.$ between two consecutive work rates $\left.<2.1 \mathrm{~mL} \cdot \mathrm{kg}^{-1} \cdot \mathrm{min}^{-1}\right)$; and (4) maximal respiratory exchange ratio $((\operatorname{RERmax})>1.1)[14,15]$.

Trunk acceleration was measured using a 'hitoe' smart clothing system (Figure 1; NTT Corp., Tokyo, Japan and Toray Industries Inc., Tokyo, Japan), which can be used to measure the heart rate and trunk acceleration [16]. This smart clothing system comprised a 'hitoe' wear, 'hitoe' transmitter, and smartphone application. An accelerometer embedded in the 'hitoe'transmitter placed on the chest measured the trunk acceleration. The sampling rate was $25 \mathrm{~Hz}$. The transmitter sent the data to a smartphone using Bluetooth Low Energy (BLE). The smartphone application was created by authors using the 'hitoe'SDK kit (NTT DOCOMO Inc., Tokyo).

\subsection{Parameters}

The intensity of the activity was assessed by the percent $\mathrm{VO}_{2}$ reserve $\left(\% \mathrm{VO}_{2} \mathrm{R}\right)$, a gold standard for the assessment of exercise intensity[17].

$\% \mathrm{VO}_{2} \mathrm{R}$ was calculated using the following equation:

$\% \mathrm{VO}_{2} \mathrm{R}=\left(\mathrm{VO}_{2}-\right.$ resting $\left.\mathrm{VO}_{2}\right) /\left(\right.$ Maximum $\mathrm{VO}_{2}$ during treadmill testing - resting $\left.\mathrm{VO}_{2}\right)$. 
The $\mathrm{VO}_{2}$ value during sitting and the maximum $\mathrm{VO}_{2}$ value during treadmill exercise testing using the Bruce protocol were used for measuring resting and maximum $\mathrm{VO}_{2}$, respectively.

Several movement quantification indices based on trunk acceleration were compared with $\% \mathrm{VO}_{2} \mathrm{R}$ in this study. The moving mean, moving standard deviation, and moving root mean square over a window of 50 samples $(2 \mathrm{~s})$ of the trunk acceleration were calculated using data for the last $2 \mathrm{~s}$ (50 data points). The norm of acceleration was calculated using the following equation:

$$
\text { Norm }=\sqrt{\left(x^{2}+y^{2}+z^{2}\right)},
$$

where $x, y$, and $z$ represent the vertical, lateral, and anterior/posterior axes, respectively.

$\% \mathrm{VO}_{2} \mathrm{R}$ and acceleration-based indices used for the analyses were averaged during the middle $1 \mathrm{~min}$ of each 3-min stage during the treadmill testing.

\subsection{Analyses}

The normality assumption was checked using the Shapiro-Wilk test. Pearson bivariate correlations were used to assess the simple relationship between $\mathrm{VO}_{2}$ and acceleration-based indices. To investigate whether the acceleration-based indices differentiate the different levels of physical activity defined in the Bruce protocol, the values of MA, MSD, and RMS, and $\% \mathrm{VO}_{2} \mathrm{R}$ as a reference, at levels 1 to 4 in the Bruce protocol were compared.

To investigate the within-subject relationships between the acceleration-based indices and $\%_{2} \mathrm{O}_{2} \mathrm{R}$ and its variability, three linear regressions were performed for each subject: values of $\%_{\mathrm{VO}} \mathrm{R}$ vs. $\mathrm{MA}, \%_{\mathrm{VO}} \mathrm{R}$ vs. $M S D$, and $\% \mathrm{VO}_{2} \mathrm{R}$ vs. RMS. Mean values and standard deviations (SD) for intercepts, slopes, and coefficients of determination were calculated for each regression.

Statistical analyses were performed using JMP11 software (SAS Institute Inc., Cary, NC, USA).

\section{Results}

First, the simple relationship with the Pearson correlation between $\mathrm{VO}_{2}$ and acceleration-based indices were assessed. Significant correlations were observed between $\% \mathrm{VO}_{2} \mathrm{R}$ and $\mathrm{MA}, \%_{\mathrm{VO}} \mathrm{R}$ and $\mathrm{MSD}$, and $\% \mathrm{VO}_{2} \mathrm{R}$ and $\mathrm{RMS}(\mathrm{r}=0.87$ and $\mathrm{P}<0.01, r=0.96$ and $\mathrm{P}<0.01, r=0.92$ and $\mathrm{P}<0.01$, respectively). Scatter plots are shown in Figure 2.

To evaluate the strength and the variety of within-individual relationship between the acceleration-based indices and $\mathrm{VO}_{2} \mathrm{R}$, the mean, $\mathrm{SDs}$ and $\mathrm{CV}$ s for the intercepts and slopes from the individual linear regression models and their coefficients of determinant were evaluated (Table 1). The averaged coefficients of determination for $\% \mathrm{VO}_{2} \mathrm{R}$ vs. $\mathrm{MA}, \%_{\mathrm{VO}} \mathrm{R}$ vs. $\mathrm{MSD}$, and $\% \mathrm{VO}_{2} \mathrm{R}$ vs. RMS were $0.89 \pm 0.05$, $0.96 \pm 0.03,0.91 \pm 0.05$, respectively. 
Table 1

Intercepts, slopes, and coefficients of determinants from the individual linear regression models

\begin{tabular}{|llll|}
\hline & MA & MSD & RMS \\
\hline y intercept & $-4.98 \pm 2.27$ & $0.27 \pm 0.07$ & $-1.00 \pm 0.34$ \\
\hline Slope & $5.47 \pm 2.20$ & $0.86 \pm 0.13$ & $1.44 \pm 0.29$ \\
\hline $\mathrm{R}^{2}$ & $0.90 \pm 0.05$ & $0.96 \pm 0.03$ & $0.91 \pm 0.05$ \\
\hline $\begin{array}{l}\text { MA, moving average of acceleration; MSD, moving standard deviation of acceleration; RMS, root } \\
\text { mean square of acceleration; SD, standard deviation; CV, coefficient of variation. }\end{array}$ \\
\hline
\end{tabular}

Then, the discriminative capacity of the acceleration-based indices in detecting different levels of exercise task were tested comparing the data of levels 1, 2, 3, and 4 in Bruce protocol. The values of MA, MSD, $\mathrm{RMS}$, and $\% \mathrm{VO}_{2} \mathrm{R}$ at levels 1 to 4 in the Bruce protocol are shown in Figure 3. The values at level 1-4 were $0.98 \pm 0.02,0.99 \pm 0.02,1.00 \pm 0.02$, and $1.06 \pm 0.04$ for MA; $0.13 \pm 0.02,0.23 \pm 0.03,0.34 \pm 0.03$, and $0.70 \pm 0.22$ for MSD; $0.99 \pm 0.02,1.01 \pm 0.02,1.06 \pm 0.02$, and $1.28 \pm 0.15$ for RMS; and $32.6 \pm 4.9 \%, 47.0 \pm 6.2 \%, 61 \pm 7.9 \%$, and $86.5 \pm 11.6 \%$ for $\mathrm{VO}_{2} \mathrm{R}$, respectively. Significant differences between levels 1 and 2 , levels 2 and 3 , and levels 3 and 4 can be observed in all indices $(P<0.01)$.

\section{Discussion}

In this study, the relationship between $\mathrm{VO}_{2} \mathrm{R}$ and the acceleration-based movement indices, MA, MSD, and RMS-calculated from the measurement of trunk acceleration using a smart clothing system-was examined. Overall, the acceleration-based indices were significantly correlated with $\mathrm{VO}_{2} \mathrm{R}$. The results of the regression analysis of each subject showed that MA, MSD, and RMS all fit the linear regressions, with MSD showing the best fit with the individual linear regressions. Using these acceleration indices, the different levels of exercise intensity defined in the Bruce protocol were clearly identified.

The overall correlation between the trunk acceleration with waist-worn accelerometer and the oxygen consumption has been shown previously $[18,19]$. The results of this study showed that this correlation is also seen between the values measured by chest-worn accelerometer and the exercise intensity estimated from the oxygen consumption that is frequently used in the exercise prescription in the rehabilitation practice[20]. In addition, we tested several indices of acceleration, such as MA and MSD-which are basic indices that represent the amplitude and fluctuation of values-and the RMS-which has been used in previous studies that quantified running using an accelerometer $[18,21]$. Among these indices, MSD exhibited the strongest correlation with $\mathrm{VO}_{2} \mathrm{R}$, and the least variability between the subjects. This may be related to the measurement of gravitational acceleration. Although the gravitational acceleration is constant, it is much larger than the dynamic component of acceleration, and a small measurement error rate may still influence the results of the measurement. Considering that the measurement of acceleration is affected by environmental conditions such as temperature [22] and that the necessity of frequent 
calibration would complicate measurement (which is the primary benefit of the accelerometer), measurement values that do not include gravitational acceleration could represent a better alternative. While MA and RMS are indices that include gravitational acceleration, MSD is an index of fluctuations from the moving average, which focuses more on the dynamic component of values. Although there may be more sophisticated methodology such as the use of autocalibration methodology to eliminate the gravitational acceleration [23], the simple solution to calculate moving standard deviation without complex data analysis can be easily applied regardless of the measurement devices. This is an advantage of the use of the MSD in the assessment of physical activity.

The correlation between the $\mathrm{VO}_{2} \mathrm{R}$ representing relative increase in oxygen consumption and the trunk acceleration is logically derived from the intensity of physical motion of the trunk. In fact, the trunk is the heaviest body segment [24, 25]; thus, its movement can largely affect oxygen consumption. Therefore, trunk movement can possibly provide more accurate measurements on exercise intensity than upper-limb movement, which varies extensively in patients with motor impairment. Although the measurement of trunk movement with a chest-mounted accelerometer may not be as easy as with wrist-worn accelerometers, the use of a smart clothing system can make it more feasible.

The acceleration indices also identified different levels of exercise tasks, which was defined by the speed and inclination of the treadmill in the Bruce protocol. This is reasonable considering that the large stride related to the high walking speed and inclination of treadmill requires a large vertical movement of the human body, and the cadence, which is the frequency of the steps, also increases to adjust to the high treadmill speed. Among the indices, MSD showed the least overlap in values between the levels 1, 2, 3, and 4 , indicating better accuracy than the other indices in describing the physical intensity of the activity. However, the variability of the values at level 4 was markedly larger in the acceleration indices than the lower levels of the exercise task. This may be because of the variety of the participants' motions during the task; for example, the participants either walked or ran at this level and some of them used a handrail to control their body posture against the high speed and high inclination of the treadmill. Therefore, the acceleration index should reflect the participants' responses to the task in addition to the level of the exercise task itself, which may well reflect the exercise intensity in the acceleration indices.

Recent advancements in measurement technologies emphasize the potential feasibility of acceleration measurement in rehabilitation settings; however, several problems can occur when employing the commonly used measurement methodology and the indices of acceleration measurement for evaluating the activities of patients with movement disorders.

Two major types of accelerometers are commercially available: wrist-worn accelerometers and body-worn accelerometers. Wrist-worn accelerometers are easy to use, and numerous studies have shown the validity of activity measurements using these devices in healthy individuals $[5,26]$. However, there is a concern regarding their usage in the case of patients with movement disorders. For patients with disabilities, the upper limb movements in daily life vary extensively for reasons such as upper-limb 
paresis or the use of walking aids; this may negate the validity of measurements provided by wrist-worn accelerometers.

As for body-worn accelerometers, the most commonly used devices are waist-worn pedometers. Although the step measurement with pedometers is widely used for activity quantification [6], the accuracy of measurements in patients with motor impairments has been questioned possibly owing to the low walking speeds, irregular movement patterns, or the use of walking aids. To utilize acceleration measurements in the field of rehabilitation, where most of the patients have motor impairment, it is necessary to develop measurement methods and indices that are resistant to variations in the motions of patients with motor impairment. On the contrary, there are several reports on chest-worn accelerometers for gait monitoring or posture monitoring [7-11]; however, the usability of chest-worn type devices for evaluating the intensity of the activity or the quantification of the activity has not been well investigated.

The present results would support the usability of the MSD of trunk movement with the chest-worn accelerometer in assessment of physical activity. Several studies have focused on heart rate-based activity monitoring using chest-strap or smart clothing monitors [27-31]. The MSD of trunk acceleration measures a similar activity; however, from a different perspective, while heart rate is a measure that reflects blood supply and is also correlated with oxygen supply, acceleration reflects actual physical movement as an output. In healthy individuals, the regression in each individual would be similar, as shown in this study. However, McGregor et al. reported that the relationship between acceleration measurements and oxygen consumption can vary with exercise experience [18]. Accordingly, the relationship between supply and output may vary more in people with motor impairment. Evaluating this relationship will expand the possibilities of activity measurement. For example, quantification of the activity in terms of both supply and output could enable the assessment of exercise efficiency; a patient with severe paresis may need more blood supply, which results in an increase in the heart rate, while performing less physical movement than a patient with mild paresis (Supplementary data). Further exploration of these methodologies may provide a meaningful and clinically viable model for the use of activity monitoring in rehabilitation settings.

The small sample size and limited variety in sample are the limitations of this study. Because of the high correlation between the acceleration-based indices (RMS and device-specific parameters) and $\mathrm{VO}_{2}$ measurements reported previously $[18,19]$, the required minimum sample size is calculated as eight (1- $\beta$ 0.95 , a 0.05 ; calculated using the sample-size-calculating software $G *$ Power, version 3.1.9.2) [32]. This might be acceptable in an experiment with young, healthy subjects; however, further investigation for applying it in population with a wider range of variety is needed. As the final goal of this study is to investigate a methodology that is suitable for measuring the activity of the patients with movement disability, the validity of using the proposed index in clinical population should be tested with larger clinical samples.

\section{Conclusions}


In this study, we compared acceleration-based trunk movement indices measured using a smart clothing system with the exercise intensity measured with an expiratory gas analysis. We found that the MSD of the trunk acceleration is highly correlated with the $\% \mathrm{VO}_{2} \mathrm{R}$. Further studies to investigate factors that influence the relationship between MSD and exercise intensity would promote the utilization of accelerometry in activity monitoring.

\section{Abbreviations}

$\mathrm{VO}_{2} \mathrm{R}$, percent $\mathrm{VO}_{2}$ reserve; $\mathrm{MA}$, mean acceleration; $\mathrm{MSD}$, mean standard deviation; RMS, root mean square

\section{Declarations}

\section{Ethics approval and consent to participate:}

The study was approved by the Medical Ethics Committee of Fujita Health University (HM17-220, approved 7th Oct. 2017). Informed consent was obtained from all subjects involved in the study. All methods were carried out in accordance with relevant guidelines and regulations.

\section{Consent for publication:}

Not applicable

\section{Data Availability Statement:}

The data collected and analyzed during the current study are available from the corresponding author on reasonable request.

\section{Competing interests:}

The research team leased the 'hitoe'system from NTT Corporation and Toray Industries Inc., which are manufacturers of the 'hitoe'system. Takayuki Ogasawara, Masumi Yamaguchi, Hiroshi Nakashima, and Shingo Tsukada are employees of the NTT Corporation. The authors declare no conflict of interest associated with this manuscript.

\section{Funding:}

This research received no external funding.

\section{Author Contributions:}

Conceptualization, M.M. and T.O.; methodology, M.M. and T.O.; software, T.O.; formal analysis, M.M., T.O., H.M. and Y.O.; investigation, M.M., H.M., Y.A. and T.S.; data curation, M.M., T.O., H.M., Y.A. and T.S.; writing -original draft preparation, M.M. and T.O.; writing-review and editing, M.Y., H.N., E.S., S.T. and Y.O; 
supervision, M.Y., H.N., E.S., S.T. and Y.O. All authors have read and agreed to the published version of the manuscript.

\section{Acknowledgments:}

The authors would like to thank Mr. Kenta Maruyama for his technical assistance with data analysis.

\section{References}

1. Cyarto EV, Myers A, Tudor-Locke C. Pedometer Accuracy in Nursing Home and Community-Dwelling Older Adults. Med Sci Sports Exerc 2004;36:205-209, DOI:10.1249/01.Mss.0000113476.62469.98.

2. Lee JA, Williams SM, Brown DD, Laurson KR. Concurrent Validation of the Actigraph Gt3x+, Polar Active Accelerometer, Omron Hj-720 and Yamax Digiwalker Sw-701 Pedometer Step Counts in LabBased and Free-Living Settings. J Sports Sci 2015;33:991-1000, DOI:10.1080/02640414.2014.981848.

3. Crouter SE, Flynn JI, Bassett DR Jr. Estimating Physical Activity in Youth Using a Wrist Accelerometer. Med Sci Sports Exerc 2015;47:944-951, DOI:10.1249/mss.0000000000000502.

4. Yang L, Lu K, Forsman M, Lindecrantz K, Seoane F, Ekblom O, Eklund J. Evaluation of Physiological Workload Assessment Methods Using Heart Rate and Accelerometry for a Smart Wearable System. Ergonomics 2019:1-12. DOI:10.1080/00140139.2019.1566579.

5. Floegel TA, Florez-Pregonero A, Hekler EB, Buman MP. Validation of Consumer-Based Hip and Wrist Activity Monitors in Older Adults with Varied Ambulatory Abilities. J Gerontol A Biol Sci Med Sci 2017;72:229-236, DOI:10.1093/gerona/glw098.

6. Elsworth C, Dawes H, Winward C, Howells K, Collett J, Dennis A, Sackley C, Wade D. Pedometer Step Counts in Individuals with Neurological Conditions. Clin Rehabil 2009;23:171-175, DOI:10.1177/0269215508098895.

7. Buckley C, Micó-Amigo ME, Dunne-Willows M, Godfrey A, Hickey A, Lord S, et al. Gait Asymmetry Post-Stroke: Determining Valid and Reliable Methods Using a Single Accelerometer Located on the Trunk. Sensors (Basel, Switzerland) 2019;20. DOl:10.3390/s20010037.

8. Hsu CY, Tsai YS, Yau CS, Shie HH, Wu CM. Differences in Gait and Trunk Movement between Patients after Ankle Fracture and Healthy Subjects. Biomed Eng Online 2019;18:26. DOI:10.1186/s12938-0190644-3.

9. Yuwono M, Su SW, Moulton BD, Nguyen HT. Gait Cycle Spectrogram Analysis Using a Torso-Attached Inertial Sensor. Annu Int Conf IEEE Eng Med Biol Soc 2012;2012:6539-6542,

DOI:10.1109/embc.2012.6347492.

10. Godfrey A, Bourke AK, Olaighin GM, van de Ven P, Nelson J. Activity Classification Using a Single Chest Mounted Tri-Axial Accelerometer. Med Eng Phys 2011;33:1127-1135, DOI:10.1016/j.medengphy.2011.05.002. 
11. Gao L, Bourke AK, Nelson J. Evaluation of Accelerometer Based Multi-Sensor Versus Single-Sensor Activity Recognition Systems. Med Eng Phys 2014;36:779-785,

DOI:10.1016/j.medengphy.2014.02.012.

12. Wang FT, Chan HL, Hsu MH, Lin CK, Chao PK, Chang Y.J. Threshold-Based Fall Detection Using a Hybrid of Tri-Axial Accelerometer and Gyroscope. Physio Measure 2018;39:105002, DOI:10.1088/1361-6579/aae0eb.

13. Bruce RA, Kusumi F, Hosmer D. Maximal Oxygen Intake and Nomographic Assessment of Functional Aerobic Impairment in Cardiovascular Disease. Am Heart J 1973;85:546-562.

14. Cunha FA, Midgley AW, Monteiro WD, Farinatti PT. Influence of Cardiopulmonary Exercise Testing Protocol and Resting Vo(2) Assessment on \%Hr(Max), \%Hrr, \%Vo(2max) and \%Vo(2)R Relationships. Int J Sports Med 2010;31:319-326, DOI:10.1055/s-0030-1248283.

15. Howley ET, Bassett DR Jr, Welch HG. Criteria for Maximal Oxygen Uptake: Review and Commentary. Med Sci Sports Exerc 1995;27:1292-1301.

16. Ogasawara T, Matsunaga K, Ito H, Mukaino M. Application for Rehabilitation Medicine Using Wearable Textile “Hitoe.". NTT Tech Rev 2018;16:6-12.

17. Garber CE, Blissmer B, Deschenes MR, Franklin BA, Lamonte MJ, Lee IM, et al. American College of Sports Medicine Position Stand. Quantity and Quality of Exercise for Developing and Maintaining Cardiorespiratory, Musculoskeletal, and Neuromotor Fitness in Apparently Healthy Adults: Guidance for Prescribing Exercise. Med Sci Sports Exerc 2011;43:1334-1359, DOI:10.1249/MSS.0b013e318213fefb.

18. McGregor SJ, Busa MA, Yaggie JA, Bollt, E.M. High Resolution Mems Accelerometers to Estimate Vo2 and Compare Running Mechanics between Highly Trained Inter-Collegiate and Untrained Runners. PLoS One 2009;4:e7355, DOI:10.1371/journal.pone.0007355.

19. Kelly LA, McMillan DG, Anderson A, Fippinger M, Fillerup G, Rider, J. Validity of Actigraphs Uniaxial and Triaxial Accelerometers for Assessment of Physical Activity in Adults in Laboratory Conditions. BMC Med Phys 2013;13:5, DOI:10.1186/1756-6649-13-5.

20. Thompson PD, Arena R, Riebe D, Pescatello LS. Acsm's New Preparticipation Health Screening Recommendations from Acsm's Guidelines for Exercise Testing and Prescription, Ninth Edition. Curr Sports Med Rep 2013;12:215-217, DOI:10.1249/JSR.0b013e31829a68cf.

21. Schutte KH, Maas EA, Exadaktylos V, Berckmans D, Venter RE, Vanwanseele B. Wireless Tri-Axial Trunk Accelerometry Detects Deviations in Dynamic Center of Mass Motion Due to Running-Induced Fatigue. PLoS One 2015;10:e0141957, DOI:10.1371/journal.pone.0141957.

22. Niu X, Li Y, Zhang H, Wang Q, Ban Y. Fast Thermal Calibration of Low-Grade Inertial Sensors and Inertial Measurement Units. Sensors (Basel, Switzerland) 2013;13:12192-12217, DOI:10.3390/s130912192.

23. van Hees VT, Fang Z, Langford J, Assah F, Mohammad A, da Silva IC, et al. Autocalibration of Accelerometer Data for Free-Living Physical Activity Assessment Using Local Gravity and 
Temperature: An Evaluation on Four Continents. J Appl Physiol (1985) 2014;117:738-744, DOI:10.1152/japplphysiol.00421.2014.

24. Dempster WT, Gaughran GR. Properties of Body Segments Based on Size and Weight. American journal of anatomy 1967;120:33-54.

25. Clauser CE, McConville JT, Young JW. Weight, Volume, and Center of Mass of Segments of the Human Body; National Technical Information Service: Springfield, VA, USA: 1969; pp. 1-112.

26. Henriksen A, Johansson J, Hartvigsen G, Grimsgaard S, Hopstock L. Measuring Physical Activity Using Triaxial Wrist Worn Polar Activity Trackers: A Systematic Review. Int J Exerc Sci 2020;13:438454.

27. Arquilla K, Webb AK, Anderson AP. Textile Electrocardiogram (Ecg) Electrodes for Wearable Health Monitoring. Sensors (Basel, Switzerland) 2020;20. DOl:10.3390/s20041013.

28. Navalta JW, Ramirez GG, Maxwell C, Radzak KN, McGinnis GR. Validity and Reliability of Three Commercially Available Smart Sports Bras During Treadmill Walking and Running. Sci Rep 2020;10:7397, DOI:10.1038/s41598-020-64185-z.

29. Fouassier D, Roy X, Blanchard A, Hulot JS. Assessment of Signal Quality Measured with a Smart 12Lead Ecg Acquisition T-Shirt. Ann Noninvasive Electrocardiol 2020;25:e12682, DOI:10.1111/anec.12682.

30. Matsuura H, Mukaino M, Otaka Y, Kagaya H, Aoshima Y, Suzuki, et al. Validity of Simplified, Calibration-Less Exercise Intensity Measurement Using Resting Heart Rate During Sleep: A MethodComparison Study with Respiratory Gas Analysis. BMC Sports Sci Med Rehabil 2019;11:27, DOI:10.1186/s13102-019-0140-x.

31. Gillinov S, Etiwy M, Wang R, Blackburn G, Phelan D, Gillinov AM, et al. Variable Accuracy of Wearable Heart Rate Monitors During Aerobic Exercise. Med Sci Sports Exerc 2017;49:1697-1703.

32. Faul F, Erdfelder E, Lang AG, Buchner AG. Power 3: A Flexible Statistical Power Analysis Program for the Social, Behavioral, and Biomedical Sciences. Behav Res Methods 2007;39:175-191, DOI:10.3758/bf03193146.

33. Chino N, Sonoda S, Domen K, Saitoh E, Kimura A. Stroke Impairment Assessment Set (SIAS) a new evaluation instrument for stroke patients. Jap J Rehabil Med 1994;31:119-25.

34. Tsukada S, Kasai N, Kawano R, Takagahara K, Fujii K, Sumitomo K. Electrocardiogram Monitoring Simply by Wearing a Shirt-For Medical, Healthcare, Sports, and Entertainment. NTT Tech Rev 2014;12:1-7

\section{Figures}



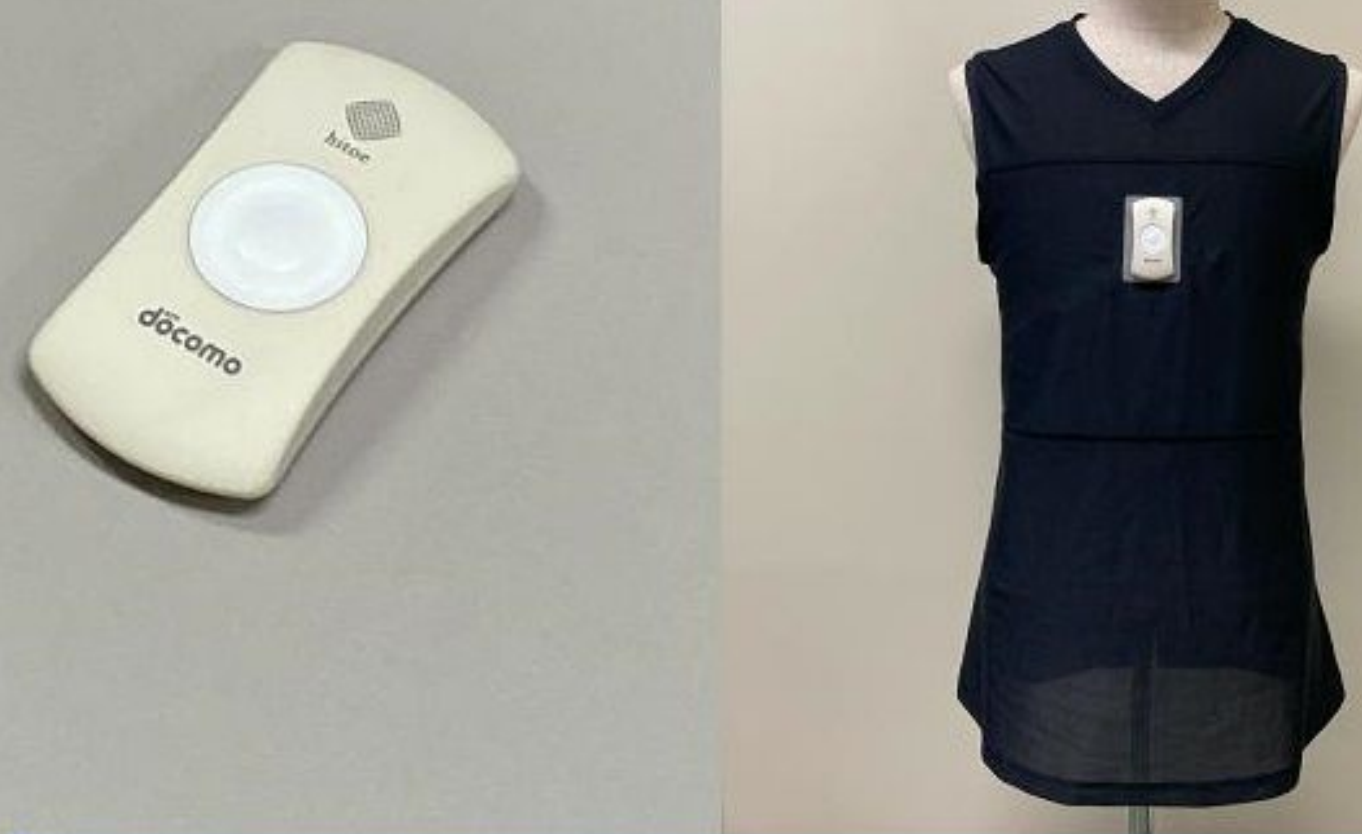

C

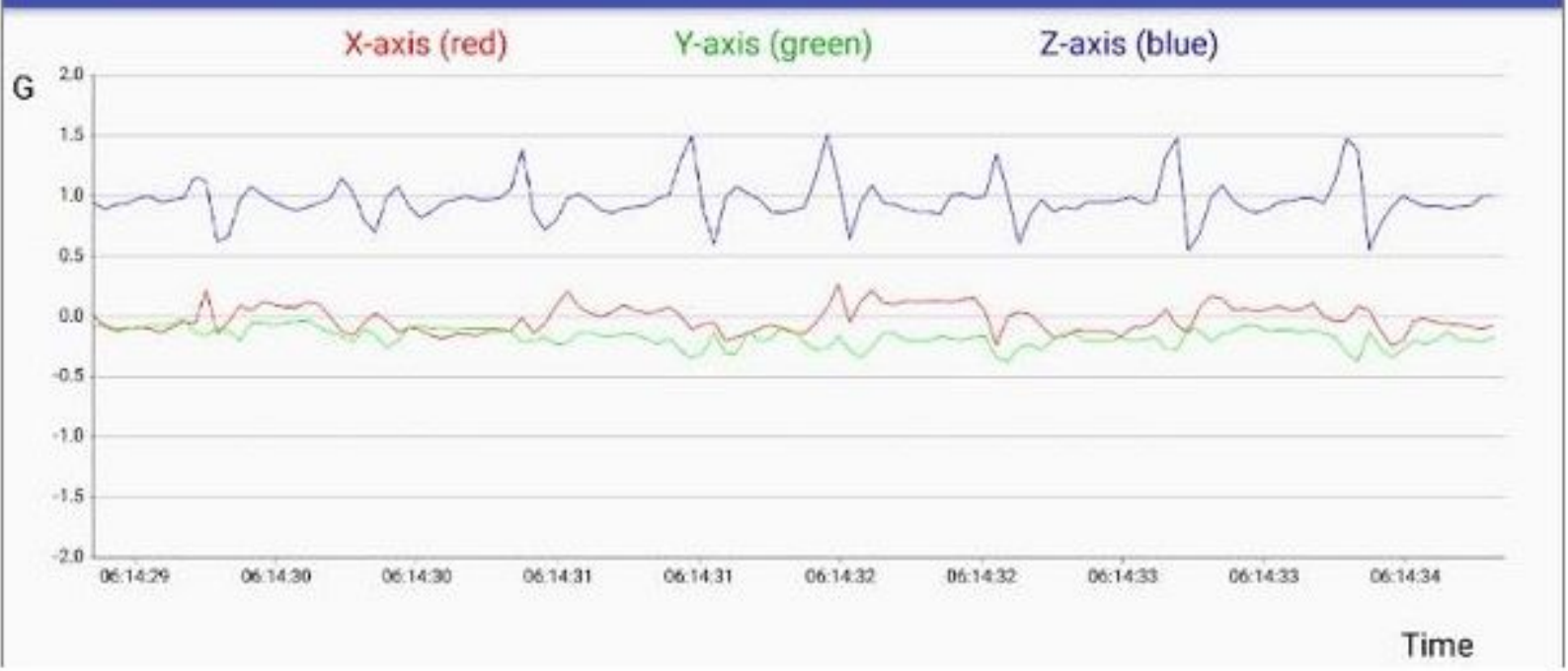

\section{Figure 1}

The 'hitoe' transmitter, the 'hitoe' wear, and the smartphone application. The 'hitoe' transmitter (A) is placed on the chest of the 'hitoe' wear (B). The data sent to the smartphone via Bluetooth can be seen on the smartphone application (C). 

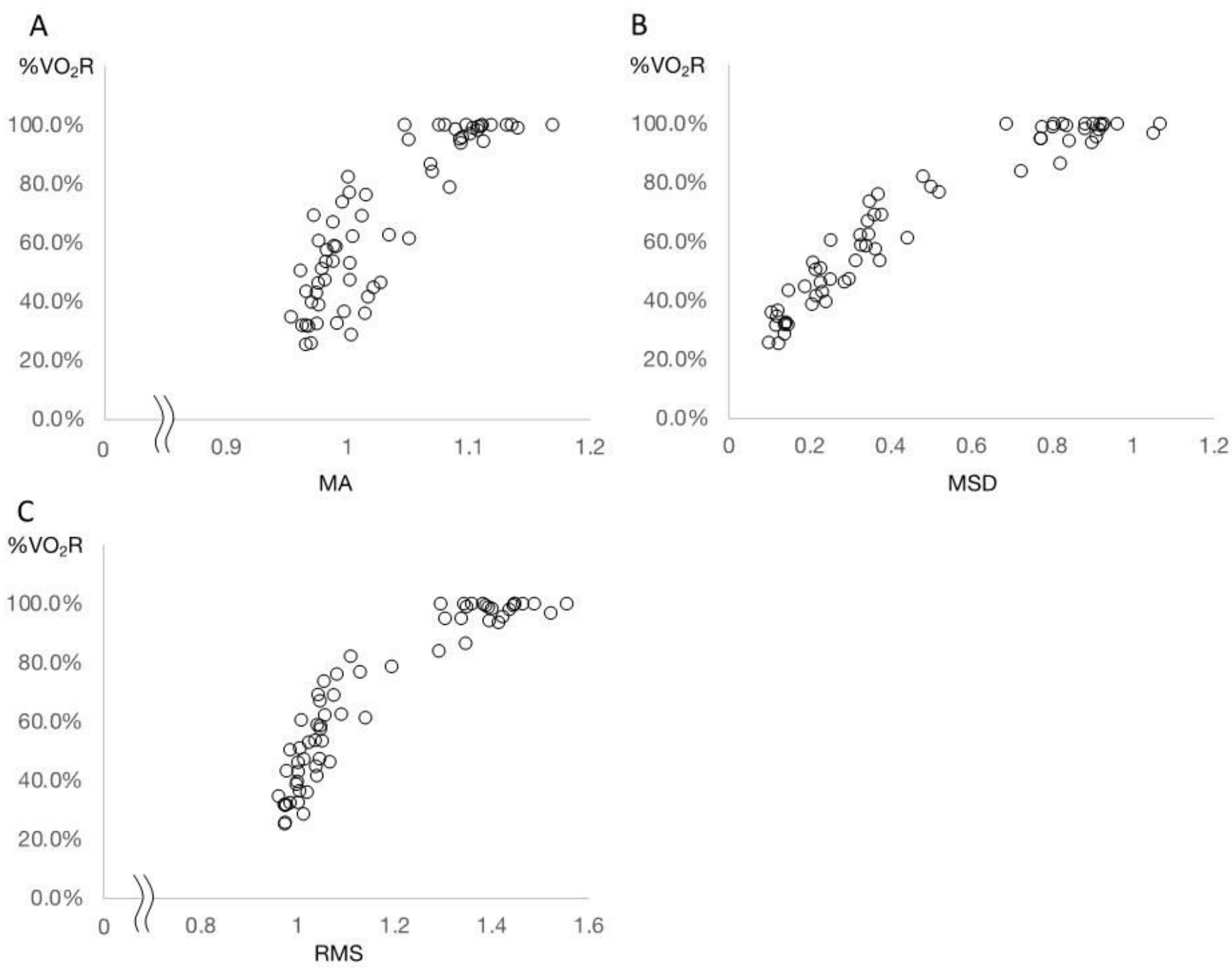

Figure 2

Scatter plot of \%VO2R vs. MA, \%VO2R vs. MSD, and \%VO2R vs. RMS. The scatter plots of acceleration indices: \%VO2R vs. MA (A: $r=0.87, P<0.01)$, \% VO2R vs. MSD (B: $r=0.96, P<0.01)$, and \%VO2R vs. RMS (C: $r=0.92, P<0.01)$. 

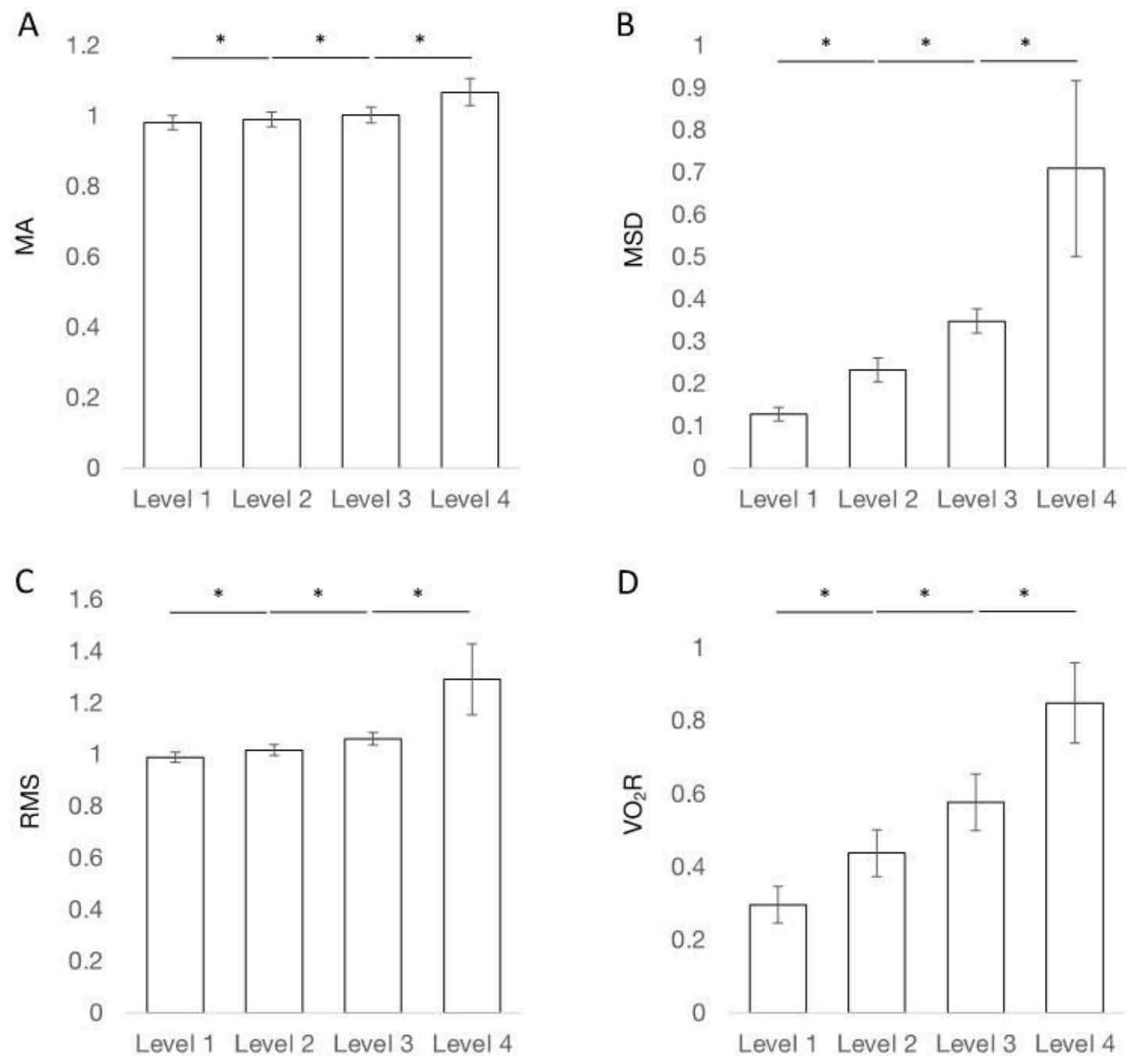

Figure 3

The acceleration indices and VO2R values in each levels of Bruce protocol The values of MA(A), MSD(B), RMS(C), and \%VO2R(D) at levels 1 to 4 are shown. ${ }^{*}<<0.01$ (Bonferrroni-adjusted).

\section{Supplementary Files}

This is a list of supplementary files associated with this preprint. Click to download.

- Supplementarymethodsandresults.docx 